EPJ Web of Conferences 41, 03024 (2013)

DOI: $10.1051 /$ epjconf/20134103024

(C) Owned by the authors, published by EDP Sciences, 2013

\title{
Photoinduced Dynamics in the Charge Density Wave Compound $4 \mathrm{H}_{\mathrm{B}}-\mathrm{TaSe}_{2}$
}

\author{
N. Erasmus ${ }^{1}$, M. Eichberger ${ }^{2}$, K. Haupt ${ }^{1}$, I. Boshoff ${ }^{1}$, G. Kassier ${ }^{1}$, R. Bimurske ${ }^{2}$, H. Berger $^{3}$ \\ J. Demsar ${ }^{2}$ and H. Schwoerer ${ }^{1}$ \\ ${ }^{1}$ Laser Research Institute, Stellenbosch University, Private Bag X1, Matieland 7602, South Africa \\ ${ }^{2}$ Department of Physics and Center of Applied Photonics and Zukunftskolleg, University of \\ Konstanz, D-78457 Konstanz, Germany, \\ ${ }^{3}$ Physics Department, EPFL, CH-1015 Lausanne, Switzerland
}

\begin{abstract}
We report on ultrafast photoindued charge density wave (CDW) dynamics in the transition-metal dichalcogenide $4 \mathrm{H}_{\mathrm{b}}-\mathrm{TaSe}_{2}$, studied with ultrafast electron diffraction. Fluence dependence of the lattice superstructure suppression show a phase transition from the commensurate to the incommensurate phase of $4 \mathrm{H}_{\mathrm{b}}-\mathrm{TaSe}_{2}$. Unusually long recovery times of perturbed charge density waves indicate th importance of a coupling between the two dimensional CDWs.
\end{abstract}

\section{Introduction}

Layered transition metal dichalcogenides $\mathrm{MX}_{2}(\mathrm{M}=\mathrm{Ta}, \mathrm{Ti} \ldots, \mathrm{X}=\mathrm{S}$, Se...) are quasi-twodimensional crystals consisting of strongly bound, 3 atom thick planar layers of X-M-X, which are weakly bound to each other by van der Waals forces along the c-axis. The metal atoms are arranged in a planar hexagonal configuration with 3 closest chalcogenide atoms above and below. In $\mathrm{TaSe}_{2}$ as one example of this class, the selenite atoms can arrange in different geometries within the layers such as octahedral or trigonal prismatic, representing different polytypes $1 \mathrm{~T}-\mathrm{TaSe}_{2}$ and $2 \mathrm{H}-\mathrm{TaSe}{ }_{2}$, respectively [1]. Due to strong electron coupling, commensurate or incommensurate charge density waves (CDW) are developed below corresponding phase transition temperatures, accompanied by periodic lattice displacements (PLD). In the pure $1 \mathrm{~T}-\mathrm{TaSe}_{2}$ a commensurate CDW/PLD of hexagonal clusters of 13 atoms with a periodicity of $\sqrt{ } 13 \times \sqrt{ } 13$ exists below $473 \mathrm{~K}$. In reciprocal space this manifests in a hexagonal superstructure in the diffraction pattern with CDW peaks of $\mathrm{a} / \sqrt{ } 13$ in between the lattice Bragg peaks originating from the underlying hexagonal periodicity with lattice constant a. The pure $2 \mathrm{H}$ polytype develops an incommensurate $\mathrm{CDW}$ only below $122 \mathrm{~K}$. The compound under consideration here, $4 \mathrm{H}_{\mathrm{B}}-\mathrm{TaSe}_{2}$, is a combination of the two: it consists of alternating layers of octahedral $(\mathrm{T})$ and trigonal prismatic $(\mathrm{H})$. Below $410 \mathrm{~K}$ the octahedral layers lock into the $\sqrt{ } 13 \times \sqrt{ } 13$ order [1], and display the corresponding diffraction patter $\mathrm{n}$ at room temperature, see Fig. 1, whereas the intermediate $\mathrm{H}$ layers act as a shield between the twodimensional CDWs.

\section{Experimental Method}

Charge density waves and their associated periodic lattice distortion can be disturbed or destroyed by

This is an Open Access article distributed under the terms of the Creative Commons Attribution License 2.0, which permits unrestricted use, distribution, and reproduction in any medium, provided the original work is properly cited. 
photons. Depending on the applied laser fluence corresponding to deposited energy, either the CDW is temporarily perturbed and will recover on a short time scale, or the transition to a higher temperature phase is driven, from which it might require longer time to return into the ordered state goverened by macroscopic cooling.

Here we investigate the photo induced dynamics of the host lattice and the CDW/PLD in $4 \mathrm{H}_{\mathrm{B}^{-}}$ $\mathrm{TaSe}_{2}$ by ultrafast electron diffraction (UED), which has proven to be a powerful technique to observe structural dynamics in crystalline material with atomic temporal and spatial resolution $[3,4]$ : a femtosecond laser pulse deposits energy into the sample, initiates the dynamic process and defines time zero; a succeeding subpicosecond electron pulse is diffracted off the sample at an optically defined and variable time $t$ and monitors the instantaneous structure of the sample. Successive recordings of diffraction patterns for varying $t$ produce an atomic-level 'movie' of the crystal dynamics. With a minimum electron pulse duration of $250 \mathrm{fs}$ at 1000 electrons per pulse, measured with an electron streak camera [4], the total temporal resolution of the experiment is $400 \mathrm{fs}$. Electron energy is $30 \mathrm{keV}$, corresponding to a deBroglie wavelength of $0.07 \AA$. The transverse coherence length being $5 \mathrm{~nm}$ is sufficient for the required spatial resolution.
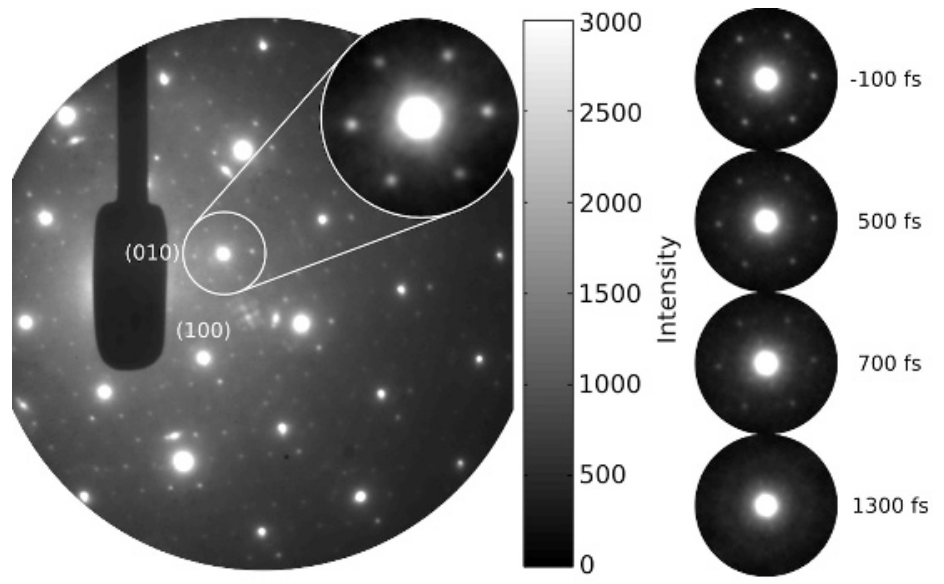

Fig. 1. Left: electron diffraction pattern of $4 \mathrm{H}_{\mathrm{B}}-\mathrm{TaSe}_{2}$ with the strong hexagonally ordered lattice Bragg reflections, and the weaker signatures of the CDW/PLD superstructure in between, again hexagonal with the $\sqrt{ } 13$ symmetry $(3 \mathbf{a}+1 \mathbf{b})$, see also insert. Right: after photo excitation, the CDW/PLD, indicated by six weak satellite reflections, disappears on an ultrafast time scale, and recovers with time constant depending on the absorbed fluence.

\section{Results and Discussion}

From the series of diffraction patterns vs. time (as in Figure 1, rigth), we determine the temporal evolution of both, the diffraction intensity into the lattice Bragg peaks as well as into the CDW signatures. Lattice- and CDW-dynamics are observed for different laser fluences: for all applied fluences, the CDW signal drops within less than a picosecond, indicating an ultrafast suppression of the superstructure order in the crystal, see Figure 2. For low absorbed fluences $\left(<2.5 \mathrm{~mJ} / \mathrm{cm}^{2}\right)$, the CDW signal is only partially suppressed and recovers within a $100 \mathrm{ps}$ time scale. This value is about two orders of magnitude larger than in related systems such as $1 \mathrm{~T} \mathrm{TaS}_{2}$ or $1 \mathrm{~T} \mathrm{TiSe}_{2}$, which miss an intermediate shielding layer. This points towards the necessity of three dimensional support for the formation of CDWs in the otherwise layered transition-metal dichalcogenides.

For absorbed fluences above $2.5 \mathrm{~mJ} / \mathrm{cm}^{2}$, where the PLD is completely suppressed by absorption of photons, the recovery time increases to several nanoseconds. The absorbed energy at threshold to full suppression corresponds to an equilibrium temperature increase in the pumped volume of about $100 \mathrm{~K}$, or from room temperature to the phase transition temperature of $410 \mathrm{~K}$ between the 
commensurate and incommensurate $\mathrm{CDW}$ phases in $4 \mathrm{H}_{\mathrm{B}}-\mathrm{TaSe}_{2}$. The fluence dependence of the recovery time almost up to the "critical" fluence corresponding to the phase transition to the incommensurate CDW phase obeys a second order character if the CDW amplitude at early times is used as the order parameter.

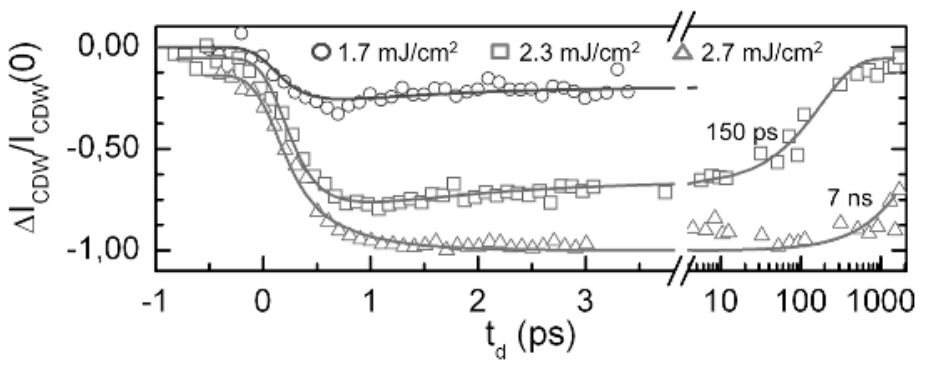

Fig. 2. CDW dynamics in the initial photoinduced suppression and the long recovery period, displayed for 3 different fluences. In the pertubative regime below $2.5 \mathrm{~mJ} / \mathrm{cm}^{2}$, the CDW recovery in the T layers of $4 \mathrm{H}_{\mathrm{B}}-\mathrm{TaSe}_{2}$ is strongly hindered by the shielding $\mathrm{H}$ layers, expressed by the long time constant of $150 \mathrm{ps}$.

Simultaneously with the suppression of the periodic lattice distortion, the lattice Bragg peaks gain intensity: upon release of the electronic correlation, the clusters of $13 \mathrm{Ta}$ atoms expand in a breathing mode towards the underlying pure hexagonal order. However, the excess energy in the electronic system rapidly couples to the crystal lattice and heats it up, and the scattered intensity decreases within less than 2 ps with the corresponding Debye Waller factor.

Both, the specific heat argument and the fluence dependence of the recovery times and CDW amplitude support the interpretation, that the laser pulses initiate a phase transition in the CDW compound tantalum diselenite, which is at least during early times of second order. Furthermore, the recovery dynamics of the CDW in the shielded polytype $4 \mathrm{H}_{\mathrm{B}}-\mathrm{TaSe}_{2}$ demonstrate the importance of a three dimensional geometry of the CDW formation in layered transition - metal dichalcogenides.

1. J. Lüdecke, S. van Smaalen, A. Spijkerman, J. de Boer, G. Wiegers, Phys. Rev. B 59, 6063 (1999)

2. B. Siwick, J. Dwyer, R. Jordan, D. Miller, Science 302, 1382 (2003)

3. M. Eichberger, H. Schäfer, M. Krumova, M. Beyer, J. Demsar, H. Berger, G. Moriena, G. Sciaini, and D. Miller, Nature 468, 799 (2010)

4. G. Kassier, K. Haupt, N. Erasmus, E. Rohwer, H. von Bergmann, H. Schwoerer, S. Coelho, and D. Auret, Rev. Sci. Instrum. 81, 105103 (2010) 